\title{
LA PRIMAVERA ÁRABE: ¿UNA CUARTA OLA DE DEMOCRATIZACIÓN?
}

\author{
Alberto Priego Moreno ${ }^{1}$ \\ Universidad Pontificia Comillas/UNISCI
}

\begin{abstract}
Resumen:
A comienzos de 2011 los países árabes del Norte de África y Oriente Medio vieron como sus poblaciones tomaban la calle para protestar frente a los regímenes autoritarios que gobiernan desde hace más de cuarenta años. Se trata de procesos de transiciones con características comunes entre si que se pueden encuadran dentro de la teoría de las olas de democratización de Samuel Huntington. Aunque no podemos preveer el futuro si que podemos afirmar que nos encontramos ante la cuarta ola de democratización.
\end{abstract}

Palabras clave: Olas de democratización, Oriente Medio, Norte de África, Revolución.

\section{Abstract:}

In early 2011 the Arab countries of Northern Africa and the Middle East have been the scenario of continuous demonstrations. They were aimed at complaining against the authoritarian regimes which have been in power for more than 40 years now. Those demonstrations may constitute the beginning of a number of transition processes with common characteristics which might be explained through the concept of "Waves of Democratization" developed by Samuel Huntington. Although we cannot make predictions for the future, we can already claim that we are witnessing the Fourth Wave of Democratization.

Keywords: Waves of democratization, Middle East, Northern Africa, Revolution.

Copyright (C) UNISCI, 2011.

Las opiniones expresadas en estos artículos son propias de sus autores, y no reflejan necesariamente la opinión de UNISCI. The views expressed in these articles are those of the authors, and do not necessarily reflect the views of UNISCI.

\footnotetext{
${ }^{1}$ Alberto Priego Moreno es Investigador UNISCI y Profesor de la Universidad Pontificia Comillas. Email: apriego@upcomillas.es.
} 


\section{Introducción y metodología de trabajo}

La democracia pueden ser concebidas bien como una escala, y así los sistemas políticos se moverían entre un grado máximo (democracia perfecta) y otro nulo ${ }^{2}$, (regímenes autoritarios) o como una dicotomía. En el segundo caso estaríamos ante una concepción binaria donde los Estados que alcanzaran un nivel adecuado en los indicadores que nos establece Robert Dahl ${ }^{3}$ en la poliarquía, serían considerados como democráticos y el resto no. Para este estudio nos basaremos en la segunda concepción aunque no desecharemos la primera ya que la línea entre una y otra es poco nítida. Sin embargo, resulta de mayor interés para la metodología elegida esta segunda visión, ya que se adapta mejor a la transitología.

A lo largo de la historia hemos visto cómo la mayor parte de los regímenes autoritarios han ido evolucionando hacia otros más democráticos a través de lo que hemos denominado procesos de transición. Por lo tanto, podemos afirmar que una transición es el paso de un régimen autoritario a otro donde se dan condiciones de competencia y participación adecuadas para ser considerado como democrático. Estos procesos pueden iniciarse a través de dos mecanismo distintos:

a) Liberalización.

b) Revuelta-Revolución.

a) Llamamos liberalización a aquellas aperturas parciales de un régimen sin que se elijan líderes gubernamentales a través de unas elecciones competitivas. Ejemplos los tenemos a millares en los procesos de transición que hemos vivido en los últimos años como puede ser la sustitución de Salazar por Caetano en Portugal, la convocatoria del referéndum de Pinochet para finales de los 80, el malogrado Tratado de la Unión de Gorbachov o más recientemente la limitación de mandatos presidenciales introducida por Raúl Castro en Cuba. Estas medidas tratan de "sacar el vapor" de esas ollas express en que, en ocasiones, se convierten los regímenes autoritarios con la única intención de acabar con la contestación popular y, en ocasiones, gracia a la presión ejercida desde el exterior.

En estos casos los gobiernos autoritarios no son capaces de satisfacer las demandas que plantea la población y por ello se ven obligados a aceptar un "second best" o tomar medidas que en un primer momento y de motu propio nunca hubieran llevado a cabo. No obstante, en ocasiones, la aceptación de estas medidas puede ser considerada como una debilidad del régimen y a medio plazo propiciar su final.

\footnotetext{
${ }^{2}$ Este es el caso de la fundación Freedom House que valora las democracias en una escala del 1 al 7 donde 1-2 son países libre, 3-5 parcialmente libres y 6-7 no libres. Disponibles en www.freedomhouse.org

${ }^{3}$ Dahl, Robert (1971): Polyarchy: Participation and Opposition. New Haven, Yale University Press.
} 
FIGURA 1: Explicación "Eastoniana" de sistemas políticos

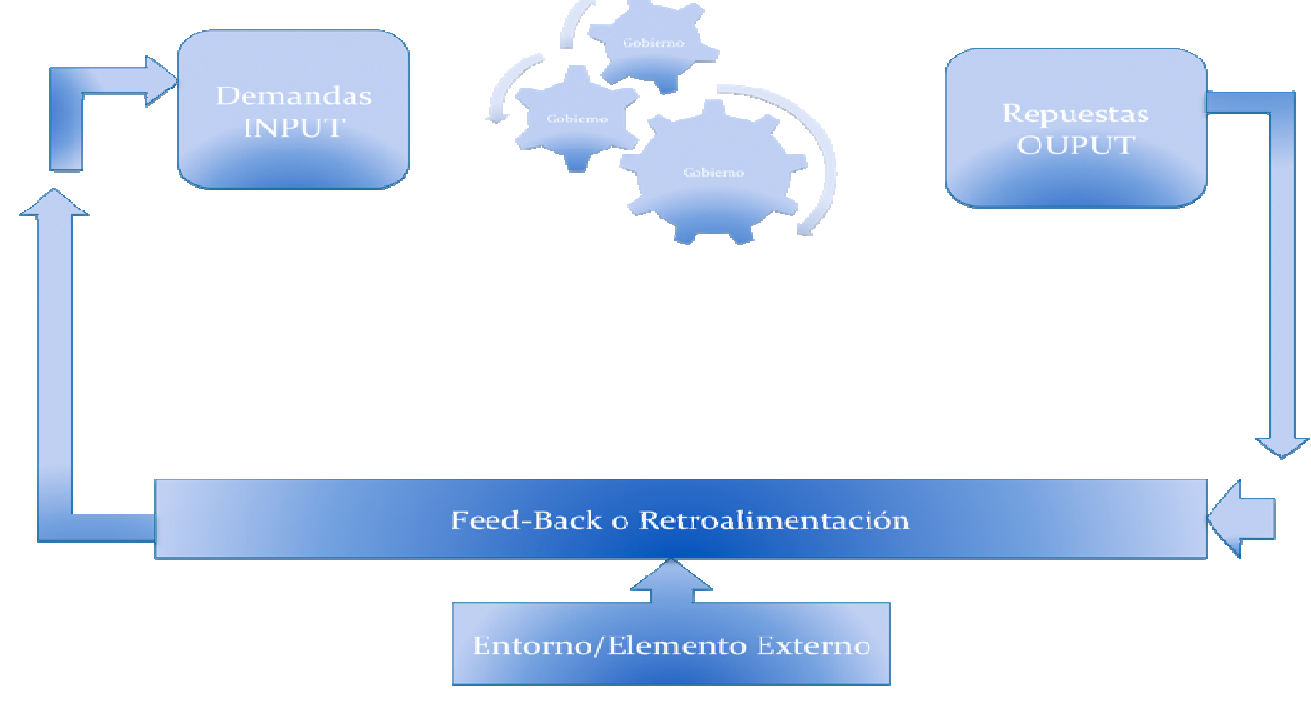

Fuente: Elaboración Propia

La población hace una serie de demandas (INPUT) que si no son atendidas por la autoridad, es decir por el gobierno, en forma de respuestas (OUTPUT) volverán a ser demandadas, pero con la carga que supone el no haber sido satisfechas con anterioridad. A esto le de debemos sumar la presión que puede ejercer la comunidad internacional (sanciones, influencia, intervenciones etc...) Si esta iniciativa no soluciona los problemas las demandas de la población perdurarán en el tiempo y nos encontramos con el segundo caso: La Revuelta.

b) La revuelta o la revolución se producirá cuando, ante la ausencia de procesos de liberalización, la presión ejercida por la población sea tal que haga caer al gobierno y en ocasiones al propio sistema político. Sin embargo, debemos diferenciar entre revuelta y revolución. Mientras que la primera podría ser considerada como cualquier alteración del orden que tiene como principal fin el derrocamiento de la autoridad, la segunda -siguiendo a Hanna Arendt- tiene dos características propias:

a) Búsqueda de un orden nuevo.

b) Ansias de libertad ${ }^{4}$.

Ninguno de los dos fenómenos garantizan que el cambio social acabe en una democracia. De hecho a veces una revuelta pasa a ser una revolución y posteriormente da lugar a una democracia ${ }^{5}$ pero en otras ocasiones las revueltas bien acaban en nada o bien acaban en otro

\footnotetext{
${ }^{4}$ Arendt, Hannah (2004): Sobre la Revolución, Madrid, Alianza Editorial pp. 50-57

${ }^{5}$ El caso de los Estados Unidos en 1776 con la Revuelta del Te en Boston que posteriormente dio paso a la Revolución Americana y a un sistema democrático como es norteamericano.
} 
tipo de régimen no democrático ${ }^{6}$. Este concepto, conocido como backsliding, no es el objeto de este trabajo ${ }^{7}$.

Volviendo al inicio, podemos decir que una transición (democrática), es el paso de un régimen autoritario a uno no autoritario. El proceso puede iniciarse bien por un proceso de liberalización y cuando este no se produce por una revuelta/revolución que puede hacer colapsar el sistema dando paso a una transición o convertirlo en otro autoritario de características diferentes. (Ver Figura 2)

FIGURA 2: Proceso de Transición a la Democracia

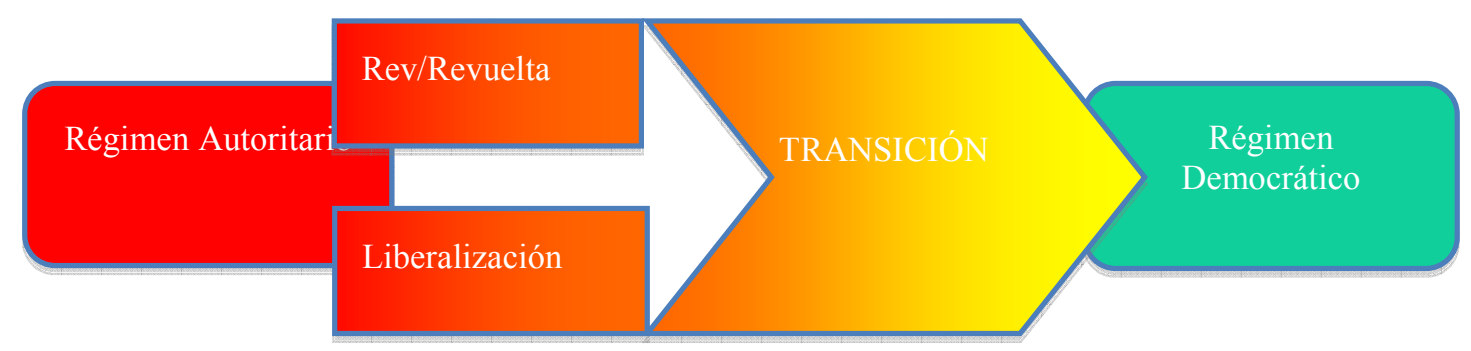

Fuente: Elaboración Propia.

En el caso de las liberalizaciones, suelen dar pie a procesos de transición que a veces son prolongados en el tiempo. Cuando esto ocurre, la ausencia de perspectiva histórica lleva a algunos autores ${ }^{8}$ a afirmar que las dictaduras se convierten en "dictablandas" o "democraduras". Sin embargo, los partidarios de concepciones más dicotómicas de la democracia -siguiendo a Samuel Huntington- creemos que la democratización es un proceso unido a la modernización al que tienden los Estados cuando incrementan su nivel de desarrollo. Por ello no cabe hablar de "dictablandas" o "democraduras" sino de regímenes democráticos o autoritarios ${ }^{9}$.

Es en este punto es donde entra el de "Ola de Democratización" que según la definición de Samuel Huntington se trata de "un conjunto de transiciones de un régimen no democrático a otro democrático que ocurren en determinado periodo de tiempo y que superan significativamente a las transiciones en dirección opuesta durante este mismo periodo ${ }^{10}$

Hay que hacer dos precisiones. En primer lugar decir que no todos los procesos de transición se enmarcan dentro de olas de democratización por lo que nos podemos encontrar

\footnotetext{
${ }^{6}$ Este sería el caso del Irán actual que vivió Revueltas como las del Tábaco o las del Pan sin mayores consecuencias o la Revolución Islámica del 1979 que sustituyó el regimen autoritario del Shah por el de los Ayatolás

${ }^{7}$ Hague, Rod and Harrop, Martin (2010):Comparative Government and Politics, Basingstoke, Palgrave p. 92

${ }^{8}$ Este es el caso de los autores que tienen una perspectiva continuista de la democracia. Es decir los que conciben un todo difuso que va desde los Estados no democráticos a los democráticos con diferentes grados.

${ }^{9}$ Para las categorías de régimen autoritario y no autoritario ver Linz, Juan (2000): Totalitarian and Authoritarian regimens, Boulder and London, Lynne Rienner.

${ }^{10}$ Huntington, Samuel (1994): La Tercera Ola. La democratización a finales del Siglo XX, Barcelona, Editorial Paidós p. 26.
} 
con procesos aislados. En segundo lugar, señalar que a cada ola le sigue una contra-ola que va en sentido contrario, es decir, fuerzas que tratan de cambiar regímenes democráticos por autoritarios $^{11}$. El autor de esta teoría, Samuel Huntington nos señala las siguientes olas de democratización:

CUADRO 1: Las Olas y Contra-olas de Democratización

\begin{tabular}{|c|c|c|}
\hline Ola /Contraola & Periodo & Estados \\
\hline Primera Ola & 18281926 & $\begin{array}{l}\text { EE.UU, Australia, Canadá Finlandia, Suecia, Suiza } \\
\text { etc... }\end{array}$ \\
\hline Primera Contra-ola & $1922-1942$ & Italia, Alemania, España, Lituania, Letonia etc... \\
\hline Segunda Ola & 1943-1962 & RFA, Japón, Italia, Jamaica, Trinidad Tobago etc... \\
\hline Segunda Contra-ola & $1958-1975$ & Birmania, Corea del Sur, Turquía, Pakistán etc... \\
\hline Tercera Ola & $1974-1990$ & $\begin{array}{l}\text { España, Portugal, Grecia, Polonia, Chequia, Hungría, } \\
\text { Bulgaria, Rumanía, etc... }\end{array}$ \\
\hline Tercera Contra-ola & $1990-2007$ & $\begin{array}{l}\text { Venezuela, Bolivia, Pakistán, Tailandia, Nicaragua } \\
\text { etc... }\end{array}$ \\
\hline Cuarta Ola & $2003-2011$ & $\begin{array}{l}\text { Georgia, Ucrania, Líbano, Irán (fallido), Azerbaiyán } \\
\text { (fallido) Egipto, Túnez ... }\end{array}$ \\
\hline Cuarta Contra-ola & & \\
\hline
\end{tabular}

Fuente: Elaboración Propia

Samuel Huntington elaboró una teoría sobre la democracia que concluía en los años 90 cuando se desquebrajó el Imperio Soviético. No obstante, a esta tercera ola, que se centró sobre todo en el sur y este de Europa, le siguió una contra-ola de regímenes autoritarios centradas sobre todo en América Latina y en menor medida en Asia. Así, llegamos al punto donde centraremos la hipótesis del trabajo: la existencia de una cuarta ola de democratización que es la que hoy estamos viviendo.

\footnotetext{
${ }^{11}$ Estas contra-olas tienen unas fuerzas que actúan para lograr sus objetivos. En la Primera Ola encontramos a los 100.000 hijos de San Luis, en la Segunda Ola a las fuerzas soviéticas que aplicaban la Doctrina Breznev y en esta Cuarta Ola las fuerzas de Consejo de Cooperación del Cooperación del Golfo que han actuado para restaurar orden en Bahrein. Para este último caso ver "A chilling account of the brutal clampdown sweeping Bahrain" The Guardian 16 de abril de 2011. Disponible en http://www.guardian.co.uk/world/2011/apr/16/bahrain-eyewitnessriot-police?CMP=twt_gu
} 
Hipótesis Principal: "Tras la caída del bloque comunista a finales de los ochenta comenzó una contra-ola autoritaria que no finalizó hasta comienzos del Siglo XXI. Sin embargo, desde el año 2003 vivimos una nueva ola de democratización que posee una serie de características propias y únicas que afecta a países de gran heterogeneidad como Georgia, Ucrania, Líbano y más recientemente Túnez o Egipto"

Para el análisis correcto de esta hipotética cuarta ola, utilizaremos una aproximación de bola de nieve ya que es el método más correcto para el análisis de los acontecimientos que estamos viviendo en el Norte de África y en Oriente Medio. La explicación se centra en que un hecho, en principio aislado, provoca la reacción en otros lugares al compartir causas profundas y éstos a su vez provocan otras reacciones similares en otros lugares.

FIGURA 3: Metodología de Bola de Nieve

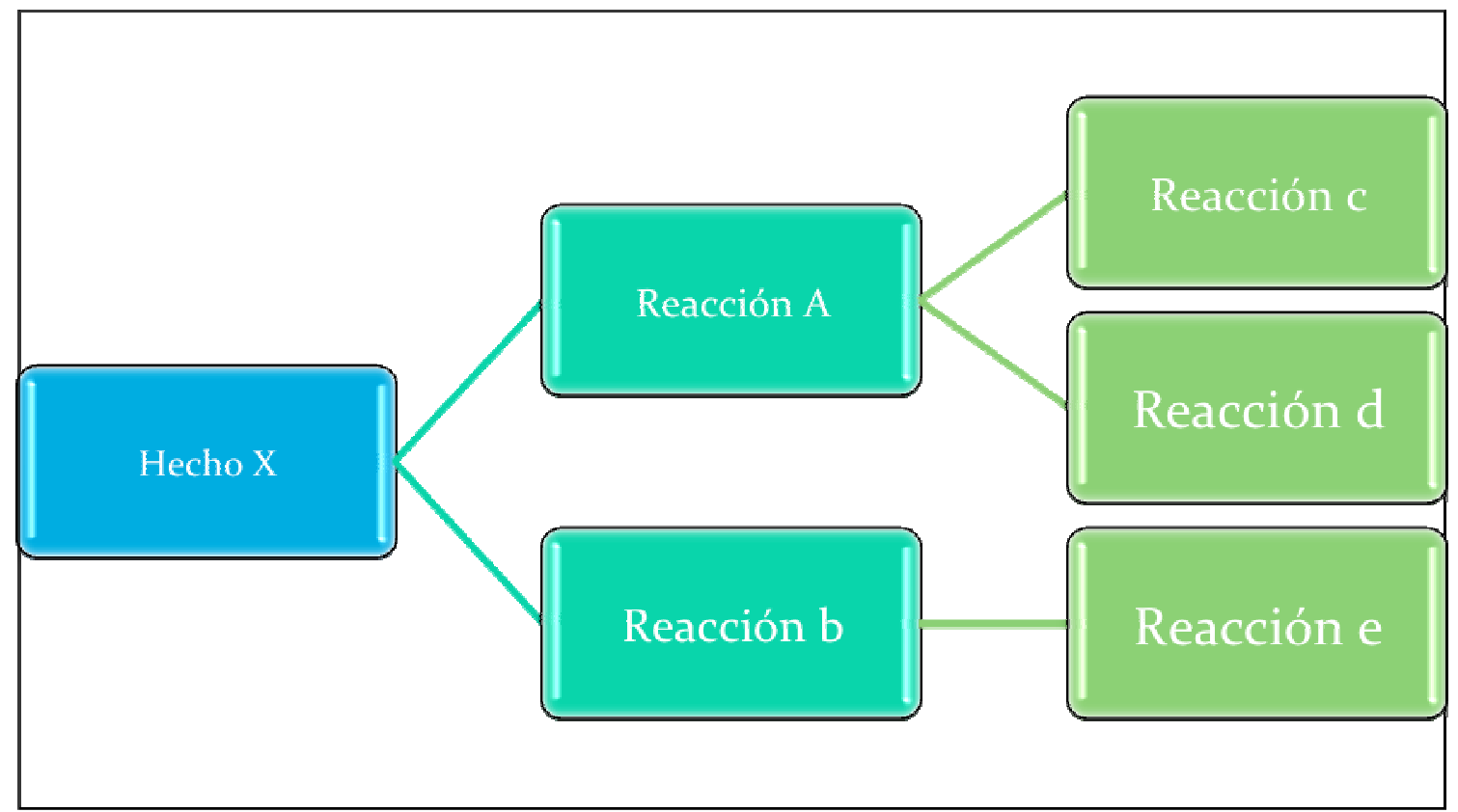

Fuente: Elaboración Propia

\section{Características de la Cuarta Ola de democratización}

Aunque Samuel Huntington no teorizó nada más allá de la tercera ola los acontecimientos que estamos viviendo en el mundo árabe bien podrían dar lugar a una cuarta ola de democratización. En esta cuarta ola de democratización encontramos dos claros grupos de Estados.

a) Los primeros serían ex repúblicas soviéticas que por su interés estratégico estaban dentro de la órbita de Rusia y que Estados Unidos pretendía acercar a su esfera de influencia a través de las denominadas revoluciones de colores. Estos son los casos de Georgia ${ }^{12}$ (Revolución de

\footnotetext{
${ }^{12}$ Priego, Alberto “Georgia ¿Otra Revolución de Terciopelo?” UNISCI Discussion Papers No. 4, Enero 2004
} 
la Rosa), Ucrania (Revolución Naranja), Kirguizistán ${ }^{13}$ (Revolución de los Tulipanes) o Azerbaiyán, donde no se llegó a desarrollar la revolución (Revolución de la Sandía ${ }^{14}$ ) Ninguno de estos casos puede ser considerado como procesos de democratización completos pero eso no elimina la opción de estar dentro de la ola de democratización. De hecho en las olas anteriores no todos los procesos analizados acabaron en un proceso de democratización completo.

b) El segundo grupo de Estados serían regímenes árabes-musulmanes que tienen algunos puntos en común. El primero de estos casos fue Irán y la frustrada Revolución Verde ${ }^{15}$ (2009) a la que seguirían los casos actuales de Egipto, Túnez, Libia, Bahrein ${ }^{16}$ o Siria. Este grupo chocaría con las ideas de aquellos teóricos que han afirmado que Islam y democracia son absolutamente incompatibles. Esta afirmación, que carece de fundamento teórico, no puede estar basada en conceptos religiosos sino que en todo caso se podría hablar de culturales, achacables a particularidades regionales más que a la propia religión. Es evidente que los países mediterráneos presentan y han presentado más dificultades para desarrollar conceptos como cultura política cívica o sociedad civil, pero en ningún caso deben ser atribuidos a una religión en concreto ${ }^{17}$.

Aunque la cuarta ola de democratización incluiría tanto regímenes de la antigua Unión Soviética como mundo musulmán en este trabajo nos centraremos únicamente en los segundos por ser más recientes y, aparentemente, más innovadores. Entre estos últimos encontramos una serie de puntos comunes que van a caracterizar esta cuarta ola de democratización y que pasamos a analizar:

a) Ausencia de liberalización.

b) Clara influencia exterior.

c) Influencia de las Nuevas Tecnologías.

\subsection{Procesos de cambio sin liberalización}

Todas y cada una de los Estados que se encuentran inmersos en este proceso de democratización tienen un punto en común que ha determinado el tipo de transición: la ausencia de procesos de liberación. Esta laguna es lo que ha provocado que en la mayor parte de los casos nos encontremos -dependiendo de los casos- ante revueltas o revoluciones (Túnez, Egipto, Libia, Siria ${ }^{18}$ etc...). De hecho, en aquellos lugares donde ha habido una

\footnotetext{
${ }^{13}$ Mikosz, David "The Kirguiz Revolution: Civil Society only works when it is real" UNISCI Discussion Papers No. 6, Mayo 2005

${ }^{14}$ Priego, Alberto "Continuidad en Azerbaiyán tras el cambio presidencial" UNISCI Discussion Papers, No. 5 Mayo de 2004

${ }^{15}$ Priego, Alberto "El Bien contra el Mal en el país de Zaratustra" El Mundo. 15-6-2009

${ }^{16}$ El caso de Bahrein es particular ya que se trata de un Estado de mayoría chíita con un gobierno sunita. Gengler, Justin "How Radical are Bahrein Shia's? The Real Source of Unrest in the Kingdom" Foreign Affairs, Mayo-Junio 2011

${ }^{17}$ Ver Priego, Alberto "Son el Islam y la Democracia incompatibles" UNISCI Discussion Papers, No.21 Octubre de 2009

${ }^{18}$ En algunos casos como Siria lo que podía haber sido una liberalización -Declaración de Beirut-Damascoacabó con mayor represión y con la detención de opositores como fueron los casos de Anwar al-Bunni o de Michel Kilo
} 
cierta, aunque tímida, liberalización - Kuwait $^{19}$ o Qatar ${ }^{20}$ con el sufragio femenino- las protestas han sido menores. Probablemente de haberse producido una liberalización previa, con la desorganización de la sociedad civil que tienen los países árabes, hoy estaríamos en otro escenario completamente diferente.

Es importante señalar la diferencia entre revuelta y revolución ya que pueden condicionar en el futuro de la transición. Por revuelta podemos entender un cambio más o menos rápido -violento o no- que tiene como principal objetivo descabezar el régimen aunque no cambiar el mismo. En el caso de la revolución, atendiendo a los estudios de Hannah Arendt, Alexis de Tocqueville y otros autores como Theda Skocpol, podríamos hablar de cuatro criterios que debe cumplir cualquier proceso de cambio social para ser considerado como revolucionario:

a) Carácter novedoso: se trata de un concepto imprescindible introducido por Hanna Arendt en el estudio de las revoluciones. En los casos los Estados que estamos estudiando no podemos decir que hayan gozado de periodos democráticos lo que les ha llevado a afirmar que para ellos el actual cambio se trata de algo totalmente nuevo. Así, podemos afirmar que "La primavera árabe" goza de este elemento que hemos señalado como imprescindible para poder hablar de revolución ${ }^{21}$ puesto que se trata de un proceso absolutamente nuevo en la región.

b) La búsqueda de una mayor libertad es el segundo de los aspectos imprescindibles para poder hablar de un proceso revolucionario. Es evidente que las protestas que han llevado a la calle a miles de egipcios, tunecinos, sirios, o yemeníes, arriesgando incluso su vida, estaban motivadas por el deseo de ser libres frente a unos regímenes autoritarios que se perpetuaban durante décadas oprimiéndolos. De hecho, todos los países donde se están produciendo estos movimiento atesoran una larga tradición de regímenes no libres tal y como muestra la escala de Freedom House.

TABLA 1: La Democracia en Oriente Medio y Norte de África

\begin{tabular}{|l|l|l|l|l|l|}
\hline & 2006 & 2007 & 2008 & 2009 & 2010 \\
\hline Bahrein & 5,5 & 5,5 & 5,5 & 5,5 & 6,5 \\
\hline Egipto & 6,5 & 6,5 & 6,5 & 6,5 & 6,5 \\
\hline Líbano & 5,4 & 5,4 & 5,4 & 5,4 & 5.3 \\
\hline Libia & 7,7 & 7,7 & 7,7 & 7,7 & 7,7 \\
\hline Túnez & 6,5 & 6,5 & 7,5 & 7,5 & 7,5 \\
\hline Siria & 7,7 & & 7,6 & 7,6 & 7,6 \\
\hline
\end{tabular}

Fuente: Freedom House ( 1 y 2 correspondería con regímenes libres, 3, 4 y 5 parcialmente libres y 6 o 7 no libres)

\footnotetext{
19 "Kuwaiti women win right to vote" BBC 17 Mayo 2005.

${ }^{20}$ En el caso de Qatar el sufragio es tanto activo como pasivo "Qatar woman re-elected in local vote" Zawaya 11 de mayo de 2011

21 "new form of activism has emerged. The Arab political space has traditionally been seen as dominated by the state through its ruling party, burocracy and security apparatus and contested, timidly or violent by Islamist movements. This no longer holds true" Fakhro, Elham and Hokayem, Emile: "Waking the Arabs" Survival, (April-May 2011) p. 22.
} 
c) Violencia (no siempre) o al menos ruptura del orden establecido. Según la doctrina marxista es inevitable la violencia para que se lleve a cabo un proceso revolucionario. Si bien es cierto que en algunos casos se está produciendo dicha violencia -Siria, Libia o Yemen- no en todos los Estados árabes que están protagonizando esta cuarta ola está presente dicho elemento. Por lo tanto, no podemos hablar tanto de violencia como de ruptura del orden establecido o quizás de una cierta distorsión de la normalidad.

d) Irresistibilidad o inevitabilidad del proceso de cambio. Este aspecto, que nació como una aportación del marxismo, ha sido recogido por Samuel Huntington en sus olas de democratización y, desde luego, las revueltas del mundo árabe no hacen más que confirmarlo. Este elemento de irresistibilidad o inevitabilidad ha sido señalado por numerosos estudiosos análisis previos a las crisis de Egipto y Túnez aunque nadie le prestó importancia ${ }^{22}$.

Podemos afirmar que los cuatro elementos que hemos seleccionado como necesarios para que se dé un proceso revolucionario están presentes en los casos de esta cuarta ola de democratización. Así, ante la ausencia de liberalización las poblaciones se han visto "obligadas" a lanzar dichos procesos. El detonante ha sido una caída brusca de las expectativas que ha resultado inasumible para una parte importante de la población que quedó escenificado en la brusca subida del precio de los alimentos y la muerte de dos jóvenes con situaciones personales terribles, Mohammed Bouazizi en Túnez y Khaled Said en Egipto.

Estos casos son similares aunque no idénticos y de hecho nos van a servir para explicar dos elementos distintos de estas revueltas. Mohammed Bouazizi era un ingeniero informático que se quemó a lo bonzo cuando su puesto de frutas fue confiscado por la policía de Ben Alí. Más allá de lo terrible del caso y de haberse convertido en un símbolo para la Revolución del Jazmín, hay que decir que el caso de Bouazizi ejemplifica como nada el detonante del proceso. No se trata tanto de la mala situación de la población como de la caída súbita y repentina de las expectativas que provoca la revuelta de la población tunecina contra el régimen de Ben Alí. Es cierto que durante años Bourgiba y luego Ben Alí han estado enriqueciéndose al tiempo que su población permanecía en la pobreza absoluta. Sin embargo en el caso de Bouazizi al igual que en Túnez no fue tanto la pobreza en la que vivía sino una caída brusca de sus expectativas de vida lo que le llevó a tomar una acción como la que tomó $^{23}$. En el caso de la población fue la séptima subida del precio de los alimentos ${ }^{24}$ de primera necesidad que unida a lo que ocurrió con Bouazizi llevó a los tunecinos a desafiar a su gobierno e incluso a derrocarlo.

Usando la Ciencia Política podríamos escenificar este cambio social con la denominada "Curva de Davies Invertida" que muestra cómo una caída brusca de las expectativas puede provocar este tipo de cambios

\footnotetext{
${ }^{22}$ Ver Taleb, Nassim Nicholas and Blyth, Mark "The Black Swan of Cairo. How Suppressing Volatility Makes the World Less Predictable and More Dangerous" Foreign Affairs, May/June 2011

${ }^{23}$ Algunos autores como Alanoud Al Sharekh han incluido una causa más remota como es el el incremento continuado de la población. Al Sharekh, Alanoud "Reform and Rebirth in the Middle East" Survival, AbrilMayo 2011, p. 51

${ }^{24}$ En Enero de 2010 el índice de la FAO que mide el precio de los alimentos (cesta de 55 productos) alcanzó los 230,7 puntos, frente a los 223,1 de diciembre. El anterior récord que databa de junio de 2008 era de 224,1 .
} 
Figura 4: Curva de J. Davies

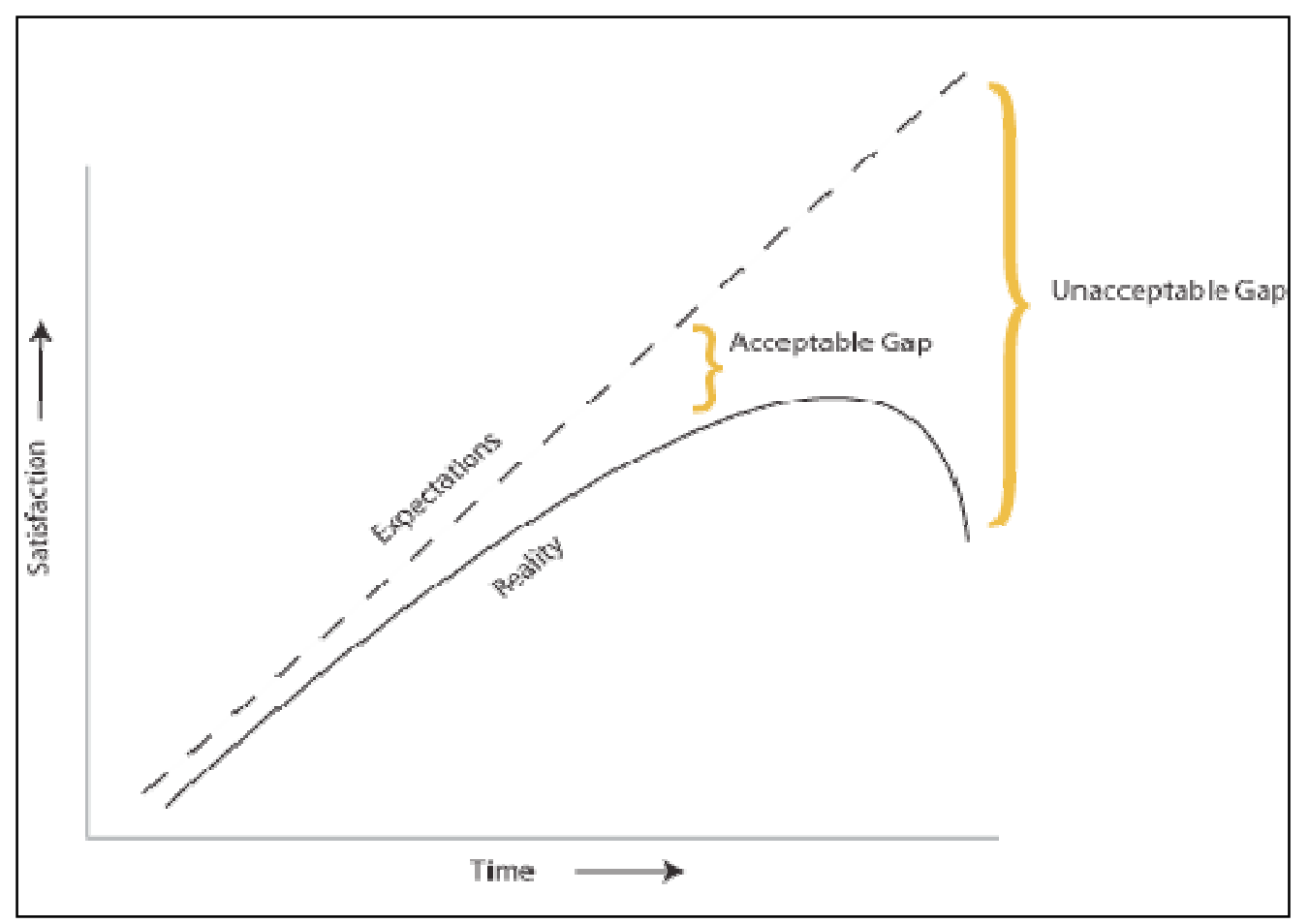

Fuente: Davies, James C. 1962. "Towards a Theory of Revolution", American Sociological Review, Vol. XXVII.

El segundo de los hechos que detonaron las revueltas fue la muerte de Khaled Said. Se trataba de un joven que fue asesinado por la policía de Mubarak pese a que la versión oficial fue que había fallecido por la ingestión masiva de drogas. La verdad es que Khaled Said era un "bloguero" con gran seguimiento en Egipto y pretendía mostrar en su blog un vídeo en el que la policía se repartía un importante alijo de drogas. Según varios testigos fue golpeado hasta la muerte. La publicación de las fotografías de su cadáver y su propagación a través de las redes sociales provocaron que la población se rebelara usando una herramienta que el régimen de Mubarak no podía controlar, las redes sociales e Internet ${ }^{25}$. Gracias a estos nuevos medios, que bien podían denominarse "sociedad civil virtual", los egipcios lograron organizarse para ocupar la Plaza de Tahir hasta la salida de Hosni Mubarak

\subsection{Importancia de las nuevas tecnologías}

Como hemos señalado en el apartado anterior la tecnología es uno de los elementos que más han influido en la organización de esta cuarta ola de democratización. El dato que mejor ilustra esta afirmación es el nombramiento por parte de Time del directivo egipcio de Google Wael Ghonim como hombre del año, por delante de personalidades académicas como el Nobel de economía Joseph Stiglitz, el fundador de Wikileaks Julian Assange o el mismísimo Presidente Barack Obama.

Wael Ghonim además de ser el responsable de marketing de Google para Oriente Medio es al mismo tiempo uno de los activistas más importantes de la Revolución Blanca, como ya se la conoce internacionalmente. Ghonim ha apoyado abiertamente a Mohammed

\footnotetext{
${ }^{25}$ Heba, Morayeb Fatma “The View From Liberation Square” The New York Times, 28 de enero de 2011.
} 
Al-Baradei ${ }^{26}$ para quien creó el perfil de Facebook y la página usada como referencia para las protestas en dicha red social. De hecho el triángulo Al-Baradei-Obama-Ghonim parece ser una de las claves de dicho proceso ya que antes de las revueltas se producen encuentros informales entre Mohammed Al-Baradei y el propio Obama. A esto le unimos que desde abril de 2010 se estrecharon las relaciones entre Ghonim y Al-Baradei.

La importancia de las nuevas tecnologías resultó crucial para el desarrollo de la protestas. Tal es así que dos días después del estallido en la Plaza de Tahir, el régimen de Mubarak trató de bloquear todo elemento digital que pudiera servir para aglutinar gente en su contra, es decir: Twitter, Facebook e incluso las redes de Blackberry ${ }^{27}$. En el caso de Egipto hay un dato que llama la atención que a pesar de tener una tasa de alfabetización muy baja (70\%) Egipto es el país de Oriente Medio con el mayor porcentaje de móvil por habitante: 8 de cada 10 egipcios tienen móvil en propiedad ${ }^{28}$.

Sin embargo, las nuevas tecnologías no han sido aplicadas sólo en Egipto; también en otros lugares y en otros momentos. Quizás el caso más claro fue la malograda Revolución Verde que agitó Irán en el verano de 2009 y donde el presidente Obama a través de discursos televisados (en Navruz) comenzó un apoyo a la población iraní con el fin hacer tambalearse al régimen de los Ayatolás. Hoy el Departamento de Estado utiliza una dirección de Twitter (@USAdarFarsi) para promover la democracia en Irán. En esta dirección se dan incluso instrucciones de cómo organizar las protestas frente al régimen autoritario que encabeza Ahmadineyab. El caso de Irán es especialmente significativo ya que se trata del Estado con mayor número de blogueros del mundo lo que supone un freno al poder autoritario del régimen.

En esta misma línea podemos decir que las primeras críticas vertidas por el Departamento de Estado sobre la represión en Túnez estuvieron centradas en la violación de la libertad de expresión en la red, lo que nos da una idea de la importancia de este medio para las revueltas y del papel jugado por los Estados Unidos en las mismas.

El uso de las tecnologías de la información es un elemento propio de esta cuarta ola de democratización pero no es nuevo. Desde hace muchos años los Estados Unidos han confiado en estos recursos para minar las bases ideológicas y sociales de los regímenes autoritarios. Buenos ejemplos son Radio Free Europe/Radio Liberty, Voice of America o Radio Nacional de Venezuela.

\subsection{Clara influencia exterior}

Tanto en los primeros procesos de esta cuarta ola (Georgia, Ucrania etc...) con en estos últimos (Egipto, Túnez, Líbano, Siria etc...) encontramos una clara influencia procedente del exterior, esencialmente de países de la UE (Reino Unido y Francia) y sobre todo de los Estado Unidos. De hecho podemos ver en esta cuarta ola de democratización una vuelta a la esencia de lo que es Estados Unidos: un Estado que tiene a la democracia y su fomento como su principal seña de identidad.

Varios son los motivos que han llevado a los Estados Unidos a promover la democracia en el mundo a través de esta cuarta ola de democratización.

\footnotetext{
${ }^{26}$ Durante mucho tiempo apareció en su perfil de Facebook la siguiente frase "Mi nombre es Wael Ghonim y apoyo publicamente a El Baredei" El Mundo 7-2-2011

${ }^{27}$ Al-Jazzera "Timeline: Egypt's Revolution" 14 de febrero de 2011.

${ }^{28}$ Al Sharekh, Alanoud, op. cit., p. 56
} 
a) El primero, citado más arriba, es la inseparable unión entre la identidad norteamericana y la democracia. De hecho, si Estados Unidos sufriera un revés autoritario desaparecería como Estado.

b) En segundo lugar, las democracias se han mostrado mucho más estables tanto desde una perspectiva interna como desde un punto de vista externo. A nivel interno el apoyo continuado a un dictador genera la animadversión de la población algo que los Estados Unidos ha conocido en primera persona en lugares como Irán, Irak, Chile o Cuba. Mientras mayor sea el apoyo a los regímenes autoritarios mayor serán las posibilidades de triunfo de un régimen de corte antioccidental. El mejor ejemplo lo tenemos en el revolucionario Ernesto "Che" Guevara: "La Revolución no puede llegar a ocurrir contra quien ha llegado al poder a través de algún voto popular y mantiene al menos una apariencia de legalidad constitucional ${ }^{29}$ " De hecho buena parte de los regímenes autoritarios del mundo tratan de dar al menos en apariencia de dar una imagen de democracia. Dos buenos ejemplos son el régimen de Mubarak o el del propio Ben Alí.

A nivel internacional parece claro que las democracias son más estables ya que son menos tendentes a la guerra que los regímenes autoritarios. Pocos son los ejemplos de democracias que atacan a otra democracia y muchos son los ejemplos de regímenes no democráticos que atacan a otros no democráticos o a democracias. Por lo tanto, la extensión o promoción de la democracia genera mayor estabilidad a nivel internacional.

Así hoy encontramos que los Estados Unidos han decidido cambiar el paradigma que regía su política exterior hasta ahora basado en la estabilidad, por otro nuevo cuyo fundamento es la democracia debido sobre todo a las razones que hemos señalado anteriormente. Para demostrar este cambio podemos acudir no sólo a los hechos sino a varios discursos pronunciados por los representantes norteamericanos de los órganos centrales su política exterior. Hagamos un breve recorrido por dichos discursos que además no entienden de partidos políticos lo que convierte a esta tendencia en una política de Estado:

a) El Presidente George W. Bush en su Segundo Discurso Inaugural habló de la necesidad de que cada Estado encontrara su propio camino hacia la democracia sin imposiciones marcando un antes y un después con su primer mandato ${ }^{30}$ donde el "spread of democracy" era interpretado como imposición de la democracia.

b) Condolezza Rice pronunció un discurso (21-6-2005) en la American University de El Cairo donde no sólo afirmaba que la democracia es el régimen político más estable sino que además reconocía que durante muchos años los Estados Unidos habían apostado erróneamente por las dictaduras frente a las democracias como principal opción de estabilidad $^{31}$. Este discurso se vio complementado por un artículo en Foreign Affairs en el que siguiendo esta línea, redefinía el Interés Nacional americano apostando por las líneas marcadas por el Presidente Bush y por ella misma unos años antes ${ }^{32}$.

\footnotetext{
${ }^{29}$ Huntington, Samuel op. cit. p. 44.

30 "America will not impose our style of government on the unwilling. Our goal instead is to help others find their own voice, to attain their own freedom, make their own way"

31 "We are supporting the democratic aspirations of all people" Disponible en "Rice speech promotes democracy in Egypt" CNN 21 de junio de 2005 http://edition.cnn.com/2005/WORLD/meast/06/20/mideast.rice/

${ }^{32}$ Rice, Condolezza "Rethinking the National Interest" Foreign Affairs, Jul/Aug 2008, Vol. 87 Issue 4
} 
c) El Presidente Barack H. Obama ha reiterado en numerosas ocasiones su confianza en la democracia como sistema más estable y por ello su deseo de continuar con la política de extensión de la democracia iniciada o, mejor dicho, recuperada por la Administración Bush ya que autores Monroe, Lincoln o Wilson ya trabajaron en esta perspectiva. La mejor muestra de este pensamiento es el discurso pronunciado ante el mundo musulmán, de nuevo en El Cairo, en el que uno de los apartados estaba dedicado precisamente a la democracia y donde incidía en los puntos destacados anteriormente.

a) La democracia no se debe imponer sino $\operatorname{promover}^{33}$ para evitar el rechazo de la población local.

b) La democracia es más estable que cualquier régimen autoritario ${ }^{34}$

c) Todos los pueblos del mundo merecen vivir en democracia ${ }^{35}$.

d) La Secretaria de Estado Hillary Clinton pronunció dos discursos con las mismas características que los anteriores. El primero de ellos en Bahrein, que a la postre sufrió revueltas, y el segundo ya en la Universidad de Georgetown.

A modo de conclusión deben ser destacadas las reacciones del Presidente Obama ante los acontecimientos que han tenido lugar en los últimos meses en Oriente Medio. Así cabe mencionarse la presión ejercida para que el Presidente Mubarak abandonara la Jefatura del Estado en Egipto o la condena del uso de la violencia por parte del gobierno en Siria ${ }^{36}$. Sin embargo, no podemos pensar que los Estados Unidos y en menor medida la UE tienen una política reactiva y que se limita a condenar los excesos de los regímenes autoritarios. Por el contrario, la sombra del Departamento de Estado es alargada y parece estar presente en las denominadas Revoluciones de Colores $^{37}$ y en la actualidad en las transiciones que están teniendo lugar en Oriente Medio. Esta afirmación puede ser completada con "el baile de embajadores" norteamericano en Oriente Medio que permanecen no solo en la zona, con independencia de la Administración que les haya nombrado, sino que están en embajadas que luego han resultado claves en las revueltas.

\footnotetext{
33 "no system of government can or should be imposed upon one nation by any other". "Obama's Speech in Cairo" Junio de 2009. Universidad Al-Azhar, El Cairo. Disponible en The New York Times http://www.nytimes.com/2009/06/04/us/politics/04obama.text.html? pagewanted $=6$

34 "governments that protect these rights are ultimately more stable, successful and secure" Idem.

35 "Those are not just American ideas, they are human rights, and that is why we will support them everywhere" Idem.

${ }^{36}$ Ver Declaración de condena de la Casa Blanca sobre la represión contra los manifestantes en Siria http://www.whitehouse.gov/the-press-office/2011/04/22/statement-president-syria

${ }^{37}$ Priego, Alberto "Georgia, op. cit.
} 
TABLA 2: Foreign Service y Oriente Medio

\begin{tabular}{|c|c|c|c|c|c|c|c|c|c|c|c|}
\hline & 2001 & 2002 & 2003 & 2004 & 2005 & 2006 & 2007 & 2008 & 2009 & 2010 & 2011 \\
\hline Siria & \multicolumn{3}{|c|}{ H. Kattouf } & \multicolumn{2}{|c|}{ M.Scobey } & \multicolumn{2}{|c|}{$\begin{array}{l}\text { Stephen A. } \\
\text { Seche }^{38}\end{array}$} & \multicolumn{2}{|c|}{ M.Corbin ${ }^{39}$} & $\begin{array}{l}\text { Maura } \\
\text { Connelly }\end{array}$ & S.Ford \\
\hline Líbano & \multicolumn{3}{|c|}{$\begin{array}{l}\text { Vincent M. Battle } \\
\text { Gerald M. Feierstein }\end{array}$} & \multicolumn{4}{|c|}{ Jeffrey D. Feltman } & \multicolumn{2}{|c|}{$\begin{array}{l}\text { Michele } \\
\text { Sison }\end{array}$} & \multicolumn{2}{|c|}{ Maura Connelly } \\
\hline Egipto & \multicolumn{4}{|c|}{ C. David Welch } & \multicolumn{3}{|c|}{ F.J.Ricciardone, $\mathrm{Jr}^{41}$. } & \multicolumn{4}{|c|}{ Margaret Scobey } \\
\hline Libia & \multicolumn{3}{|c|}{ Sin Relaciones Diplomáticas } & \multicolumn{3}{|c|}{ Greg L. Berry ${ }^{42}$} & \multicolumn{2}{|c|}{ Ch.O. Cecil ${ }^{43}$} & \multicolumn{3}{|c|}{ Gene A. Cretz } \\
\hline Bahrein & \multicolumn{3}{|c|}{ Ronald E. Neumann } & \multicolumn{4}{|c|}{ William T. Monroe } & \multicolumn{4}{|c|}{ J. Adam Erel } \\
\hline Túnez & $\mathrm{McPh}$ & nDem & \multicolumn{4}{|c|}{ William J. Hudson } & \multicolumn{3}{|c|}{ Robert F. Godec } & \multicolumn{2}{|c|}{ Gordon Gray } \\
\hline Yemen & \multicolumn{3}{|c|}{ Edmund James Hul } & \multicolumn{4}{|c|}{ Thomas C. Krajeski ${ }^{45}$} & \multicolumn{2}{|c|}{$\begin{array}{l}\text { Stephen A. } \\
\text { Seche }\end{array}$} & \multicolumn{2}{|c|}{ Gerald M. Feierstein } \\
\hline
\end{tabular}

Fuente: Elaboración Propia usando datos del Departamento de Estado

Algunos nombres se repiten como Stephen Seche quien además de ser Encargado de Negocios Ad Interim en Siria y Ministro Consejero ha desempeñado el cargo de Jefe de Misión en Yemen. En su paso por Washington fue Director de Office for Egypt and Levant Affairs desde donde se diseña la política hacia Egipto. También cabe destacar que pasó dos años en Túnez estudiando árabe. Algo parecido ocurre con Margaret Scobey que ha pasado por las embajadas de Siria y Egipto donde en la actualidad ejerce de Jefa de Misión. El que ahora es embajador en Yemen estuvo destinado en la Embajada de Líbano. Por último destacar a Maura Connelly que al igual que Scobey ha pasado por Egipto y Siria, dos de los Estados donde se están viviendo revueltas contra los regimenes autoritarios. En definitiva podemos ver que hay una serie de diplomáticos que repiten en unos cargos claves para poner en práctica la política norteamericana hacia Oriente Medio que marca el Departamento de Estado. Este mismo fenómeno ya ocurrió en la antigua URSS cuando se produjeron las conocidas como Revoluciones de Colores con nombres como Stanley Escudero o Reno L Harnish.

De lo que estamos seguros es que Washington parece haber aprendido la diferencia entre promoción e imposición de la democracia. Si bien es cierto que el primer caso puede resultar de gran interés, en el segundo aun cuando las intenciones sean buenas solemos encontrarnos con la oposición de la población. El más claro ejemplo es Irak. Quizás la mejor expresión de la estrategia norteamericana ante las revoluciones del mundo árabe la

\footnotetext{
${ }^{38}$ Encargado de Negocios Ad Interim después de retirar al Embajador tras el asesinato de Rafic Hariri

${ }^{39}$ Encargado de Negocios Ad Interim

${ }^{40}$ Encargado de Negocios Ad Interim

${ }^{41}$ Enviado a Turquía con rango de Embajador

${ }^{42}$ Encargado de Negocios Ad Interim

${ }^{43}$ Encargado de Negocios Ad Interim

${ }^{44}$ Encargado de Negocios Ad Interim

${ }^{45}$ Estuvo en la Embajada del Cairo entre 1992-1997
} 
encontremos en la frase de la Embajadora Norteamericana en El Cairo en su reunión con AlBaradei:

"The U.S. is interested in a political change in Egypt, but that the US government won't dictate the path which Cairo must follow ${ }^{46, "}$

\section{El futuro incierto}

Los acontecimientos que estamos viviendo en Oriente Medio no son más que el inicio de varios procesos de transiciones políticas. Aunque hay diferentes velocidades y por lo tanto estamos en fases diferentes aún nos quedan las más complicadas. El derrocamiento del tirano por sí mismo -cuando este se ha producido- no garantiza la consecución de la democratización. Así podríamos representar las fases de la transición para hacernos una idea de donde se encuentran estos Estados y el camino que les queda aún por recorrer:

FIGURA 5: Las Transiciones Políticas por fases

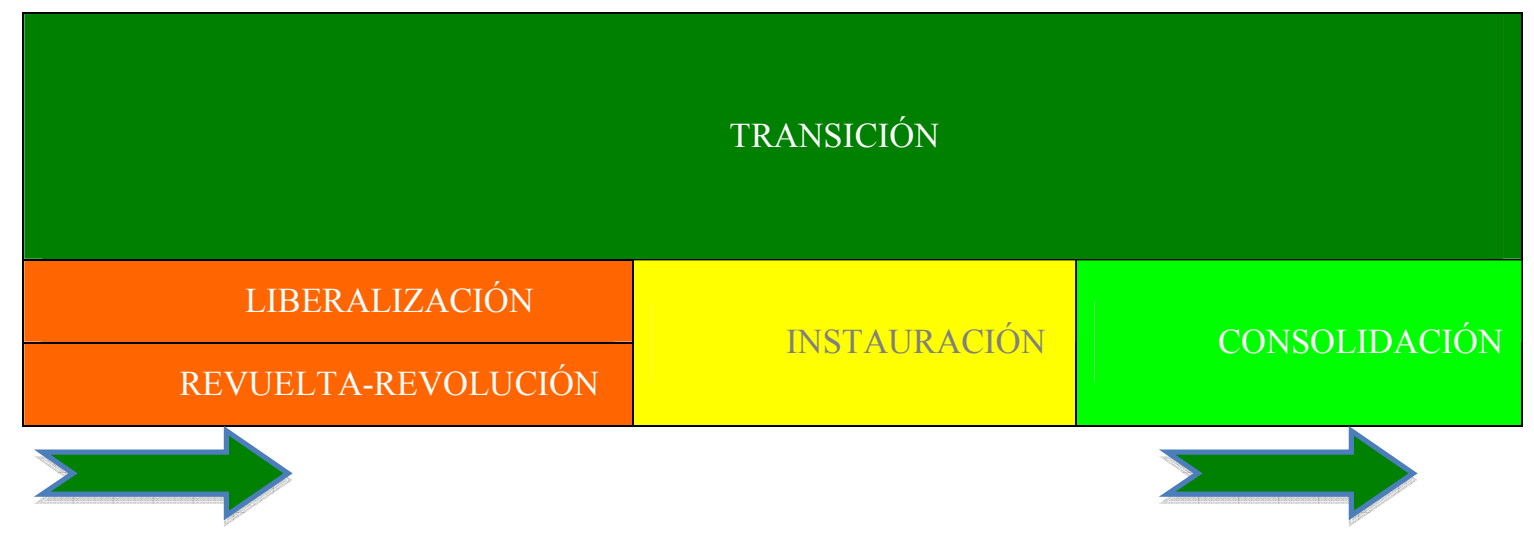

Fuente: Elaboración Propia

Sin embargo, el hecho de que hayan sido reemplazados algunos de los Presidentes de los países árabes donde se han producido rebeliones no quiere decir que se haya alcanzado la democratización. En la actualidad Egipto, Yemen y Túnez se encuentran en la fase de instauración lo que por el momento genera gran incertidumbre. Otros Estados como Siria o Libia están aún en la fase anterior, y en todo caso, todos tienen por delante un largo camino hacia la democracia. Por ello, podemos destacar una serie de problemas que tendrán que afrontar los países árabes en el futuro, unos que son comunes a todos los procesos de transiciones políticas y otros exclusivos del mundo árabe-musulmán.

YeNetNews.Com 2 de febrero de 2011. Disponible en http://www.ynetnews.com/articles/0,7340,L4022483,00.html 


\subsection{Problemas para la consolidación y obstáculos entre Islam y democracia}

Tras el momento de plenitud y de exaltación que llegó con la expulsión de los tiranos del poder -esencialmente Mubarak, Ben Alí y Saleh- los Estados se encuentran con la problemática de tener que organizar una transición. El primer paso ha sido la convocatoria de unas elecciones que sirva para elegir unas instituciones legítimas ${ }^{47}$. Sin embargo, estas democracias emergentes se encuentran con algunas necesidades o ausencias algunas propias de ser países en transición y otras propias de ser países musulmanes. Hagamos un análisis de ambas problemáticas:

\subsubsection{Ausencias de un país en transición y del mundo árabe-musulmán}

Uno de los mayores estudiosos del Islam ha sido Leonard Binder quien desarrolló el concepto de "cluster of absences" destacando aquellos aspectos que se echaban de menos en los países musulmanes para poder desarrollarse como democracias ${ }^{49}$. Si a esto le sumamos los problemas que se generan cuando se afronta cualquier proceso de instauración de la democracia vemos que son muchas las dificultades que tendrán que superar los pueblos de Oriente Medio.

a) Ausencia de experiencias previas: Cuando afrontamos procesos de democratización y no de redemocratización nos encontramos sin referencias claras o sin lecciones aprendidas como ocurrió en otros lugares como Chile, Argentina o algunos Estados de Europa del Este. En el caso de los países que estamos analizando este es uno de los principales problemas pero también es una de las principales bazas ya que no tiene rémoras del pasado ni sistemas que volver a aplicar y por lo que se podrá empezar ex novo atendiendo a las verdaderas necesidades políticas y sociales de la población.

La ausencia de experiencias previas no nos puede llevar a caer en un determinismo histórico que afirme que los Estados Musulmanes nunca serán regímenes democráticos. Este tipo de determinismos ya fueron aplicados a otros Estados, como Alemania o Italia ${ }^{50}$, donde la experiencia nos ha mostrado su falta de validez.

b) Ausencia de sociedad civil: A pesar de los esfuerzos llevados a cabo por los Estados Unidos (Fulbright, USAID etc...) los países de la cuarta ola, al igual que sus predecesores, adolecen de una importante carencia de sociedad civil. Poco a poco, los países de la tercera ola van superando este problema que impide que el poder tenga límites más allá de los establecidos en el sistema político.

En el caso de los países árabes este es un problema aun más grave ya que se denota un desequilibrio claro entre las esferas estatal y civil a favor de la primera. Este problema ha sido señalado por los Orientalistas y los Neorientalistas como uno de los principales obstáculos que tienen que superar los países árabes para democratizarse. De hecho, las únicas muestras

\footnotetext{
47 Túnez celebrará elecciones a Asamblea Constituyente el 24 de julio de 2011 y Egipto Presidenciales en Septiembre de 2011.

48 Binder, Leonard (1988): Islamic Liberalism: A Critique of Development Ideologies. Chicago and London. University of Chicago Press. p. 225.

${ }^{49}$ Binder señala una serie de carencias en el mundo árabe-musulmán entre las que podíamos destacar la ausencia del sentimiento de ciudadanía, de cultura política o de exigencias a la autoridad.

50 "the rise of fascismo in Italy as clear proof that democracy was a special local growth wich could not be guaranteed to acclimatize itself in alien soil (...) create a liberal Germany, as most emigants want to do, is likewise impossible" Sharansky, Natan and Dermer, Ron (2003): The case for Democracy. The Power of Freedom to overcome tyranny and terror, New York. Perseus Book. pp. 24-25.
} 
de sociedad civil hasta esta cuarta oleada han sido los Hermanos Musulmanes, Hamás, Hezbollah etc... Este punto será analizado al final de este apartado.

c) Ausencia de cultura política: Los regímenes autoritarios pueden reaccionar de tres modos ante la cultura política: ignorándola, manipulándola o transformándola. En el caso de los países de la cuarta ola la opción es la primera ya que el régimen no busca convencer a la población sino mantener dentro de su zona de interés usando todos los medios posibles y entre ellos, claro está, la represión ${ }^{51}$.

En principio se trata de regímenes donde la seguridad del régimen está por encima de la seguridad de la población y con un fuerte apoyo en los militares (Libia, Siria, Egipto...) De hecho, en el momento en que el régimen pierde este poder de coerción no tiene posibilidad de sobrevivir como ha ocurrido en Egipto cuando Mubarak ha perdido el favor de los militares. De hecho las fuerzas de seguridad en Egipto han resultado fundamentales en la caída de Hosni Mubarak ya que su inacción permitieron las concentraciones en Egipto en la famosa Plaza Tahir.

Una vez ha caído el dictador el problema es para la población que carece de cualquier tipo de cultura política democrática. De hecho su cultura política es generalmente de siervo, y con eso hay que construir una democracia. Este será otro de los obstáculos que tendrán que afrontar estos Estados en el futuro cercano.

En este caso, en las sociedades musulmanas apreciamos una falta de separación entre las esferas privadas (ibadat) y públicas (mummadat). Por ello resulta difícil tanto el desarrollo de una verdadera sociedad civil como de una cultura política democrática o cívica ${ }^{52}$.

d) Ausencia de grupos organizados y emergencia de los grupos musulmanes radicales: A1 igual que ocurrió en Europa del Este tras la liberación de los nazis y en Irán en 1979, las sociedades que salen de un régimen de terror se encuentran prácticamente desorganizadas. Solamente aquellos grupos que se mueven cómodamente en la clandestinidad-los comunistas en Europa del Este y los islamistas en Irán- son capaces de presentarse como la única alternativa viable y organizada.

Los Estados de esta cuarta ola no son una excepción, ya que tanto los Hermanos Musulmanes en Egipto como Nahda o el Partido del Renacimiento Islámico en Túnez se están mostrando como las opciones más preparadas para alcanzar el poder en las futuras elecciones. De hecho, en Túnez, los Islamistas ya se han hecho con 8 de los 9 puestos en disputa en la Asociación de los Jóvenes Abogados los que demuestra que, ante la desorganización, los auténticos vencedores pueden ser los más organizados, los Islamistas.

Sin embargo, algunos autores como Elham Fakho y Emile Hokayem aseguran que los radicales están teniendo un papel muy limitado en las transiciones y que los propios Hermanos Musulmanes no han podido tener el protagonismo que les hubiera gustado tener ${ }^{53}$. La situación es aún más compleja ya que en la región que estamos estudiando las líneas de conflicto existentes en el sistema social no permiten organizar agrupaciones políticas, y la acción de la dictadura ha provocado la atomización de la sociedad haciendo casi imposible la reunión de un grupo de personas en torno a un conjunto de ideas. La situación en Egipto y en Túnez recuerda peligrosamente al Irán Revolucionario donde aunque inicialmente no era

\footnotetext{
51 “We rule because we rule' remains the implicit message” Hague, Rod and Harrop, Martin, op. cit., p. 130

${ }^{52}$ Ver Priego, Alberto "¿Son Islam, op. cit., p.45

${ }^{53}$ Fakho, Elham and Hokayem, Emile "Waking the Arabs" Survival, Vol 53 No. 2, May-June 2011 p. 23.
} 
favorable al Estado Islámico, al final ante la falta de organización acabó en manos de los Ayatolás.

\section{Conclusiones}

A modo de conclusión podemos decir que los países de la antigua URSS y ahora los países del Norte de África y Oriente Medio están protagonizando la que hemos denominado "Cuarta Ola de Civilización". Esta tiene una serie de características propias como son la ausencia de liberalización, el uso de las nuevas tecnologías para suplir algunas carencias como la sociedad civil o la falta de grupos políticos organizados provocada por los años de represión. Esta ausencia de liberalización ha provocado revueltas que en algunos casos podrían ser consideradas como "Revoluciones" ya que cumple con los criterios utilizados por la ciencia política para designar a este tipo de procesos.

Así mismo podemos apreciar una clara influencia exterior que busca, para evitar antiguos fracasos, la promoción y nunca la imposición de la democracia. Por ello, los Estados Occidentales con Estados Unidos a la cabeza están tratando de promover la democracia en la región con un fin claramente egoísta y pensado a largo plazo: las democracias son mucho más estables ya que a nivel global no hacen la guerra y estables ya que un dictador acaba cayendo y la población se vuelve contra aquellos Estados que le han apoyado. Además, si los gobernantes están sometidos a procesos electorales habrá medidas que nunca tomarán, como la represión indiscriminada o la realización de cierto tipo de alianzas con otros Estados no democráticos como China o Rusia.

Sin embargo no podemos decir que haya terminado el proceso ya que por un lado podemos decir que existen varias de velocidades de cambio. El método de bola de nieve nos dice que todavía hoy continúan las reacciones en cadena en otros Estados. De hecho, incluso estas van más allá de la región y hoy encontramos importantes revueltas en Tayikistán, Azerbaiyán o Uzbekistán lo que prueba la homogeneidad de esta cuarta ola a pesar de las diferencias. Podríamos escenificar esta cuarta ola de la siguiente forma:

FIGURA 6: La $4^{\text {a }}$ Ola de Democratización

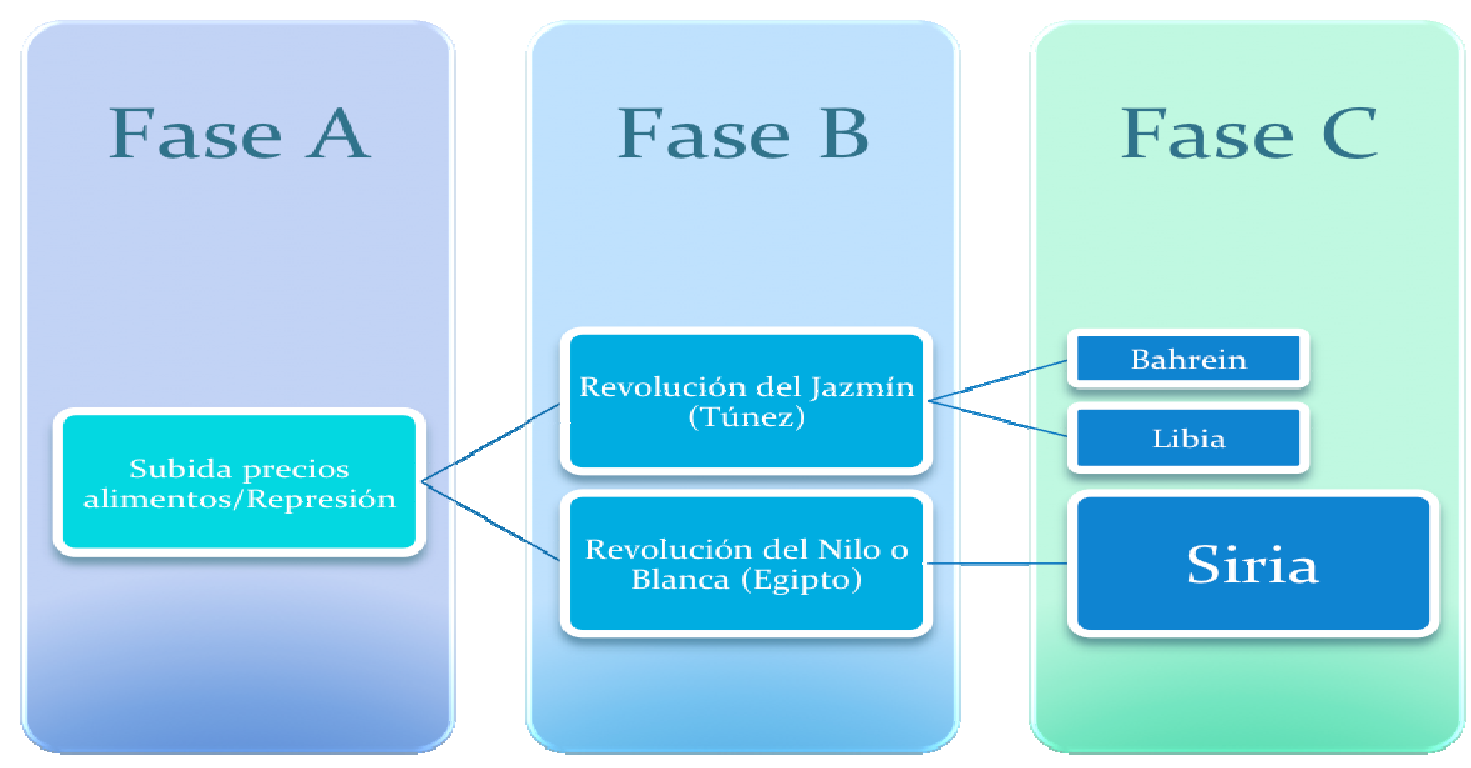

Fuente: Elaboración Propia 
Uno de los más perjudicados por las revueltas ha sido Al-Qaeda tal y como pusieron de manifiesto las declaraciones de Al-Zawahiri al afirmar que la democracia tan sólo puede ser no religiosa y por ello no es legítima ${ }^{54}$. Así, si se lograra alcanzar una democratización de los países árabes, Al-Qaeda se quedaría sin uno de sus principales "caladeros de yihadistas" y el islamismo dejaría de ser la única solución frente a los regímenes autoritarios del mundo árabe ${ }^{55}$.

Por último, decir que la caída de alguno de los líderes autoritarios que dominaban el espectro político del Norte de África y Oriente Medio, no es más que el comienzo de un proceso de transición incierto y complicado que puede llevar, incluso, a los islamistas al poder. Si se hicieran con el poder, quizás este sería el punto en el que por primera vez tendrían que hacer frente al poder del pueblo y dar soluciones a los problemas reales de los ciudadanos y que estos les pudieran juzgar por lo que hacen y no sólo por lo que dicen. Pero el ejemplo de Irán, convertido en una dictadura policial, no permite hacerse grandes ilusiones. En definitiva, la evolución será diferente en cada uno de los Estados y la prueba son los casos de Egipto y Libia donde no son comparables los acontecimientos que se han ido desarrollando. Mientras que el país del Nilo tiene marcada una hoja de ruta, Libia se ha convertido en campo de batalla donde la población se enfrenta en una guerra civil que no tiene fácil solución.

Por ello, podemos decir que nos encontramos ante una cuarta ola de democratización que dentro de unos años tendrá su contra-ola, pero cuyo resultado podría ser una mayor democratización en el mundo.

\footnotetext{
${ }^{54}$ Fakhro, Elham and Hokayem, Emile, op. cit., p. 26.

${ }^{55}$ Ante la falta de posibilidades los islamistas suelen responder con la frase "El Islam es la Solución"
} 